\title{
Structural imaging of a thick-walled carbon microtubule
}

\author{
Michael Bretz ${ }^{*, a}$, Brian G. Demczyk ${ }^{b}$, Luqun Zhang ${ }^{\text {a }}$ \\ ${ }^{a}$ Department of Physics, University of Michigan, Ann Arbor, Michigan 48109, USA \\ ${ }^{\mathrm{b}}$ Electron Microbeam Analysis Laboratory, University of Michigan, Ann Arbor, Michigan 48109, USA
}

Received 6 January 1994; manuscript received in final form 4 March 1994

\begin{abstract}
We analyze the structure of a thick-walled carbon microtubule based on direct electron beam imaging of the graphitic cylinders comprising the fiber. Clearly resolved six-fold symmetry of basal planes overlying the fiber core indicate zero overall fiber helicity and alignment of individual cylinders. Sidewall measurements calibrated from the $\{10 \overline{10}\}$ core fringes show uniform spacings of about $0.375 \mathrm{~nm}$, which are larger than those reported for other microtubules or for crystalline graphite $(0.335 \mathrm{~nm})$. Short zones of local $3^{\circ}$ helicity are observed along the fiber. Structural transitions which alter the helicity are characterized by extra atomic planes and other defects, including nested sub-tubules. We discuss implications for the fiber's growth and electrical properties.
\end{abstract}

Carbon microtubules are the thinnest and most perfectly formed carbon fibers known. Discovered recently as a byproduct of fullerene production [1], they consist of concentric graphitic cylinders of micron length having nanometer scale cores, along with a helical structure and variably shaped endcaps [2]. Procedures have been developed to produce multiwall [3] and single wall fibers [4]. Most previous electron microscopy work has relied on interpreting whole-fiber electron diffraction patterns to ascertain the fiber helicity [1]. Recently Zhang et al. [5] have performed direct imaging of fiber graphitic sheets to determine their structural properties. They reported the frequent occurrence of both non-helical tubes and tubes having cylinders of various helicities, and performed computer modeling to confirm

\footnotetext{
* Corresponding author.
}

image feature interpretations. We use direct imaging techniques and local area fast Fourier transforms (FFT) to analyze a unique microtubule. Unlike other tubules whose individual cylinders can have different helicity, ours changes axially from zero net helicity to low helicity via structural transitions within the tubule. We show that the growth is concentric rather than spiral, discuss local defects and assess the electrical implications for the fiber.

Microtubules were grown by sustaining a DC electrical arc in the gap between two graphite rods $(99.9995 \%$ pure, $6.15 \mathrm{~mm}$ diameter) at a nominal $110 \mathrm{~A}$ in 320 Torr of helium gas (prepurified, liquid nitrogen trapped). Material from the cathode end was scraped and sonicated in isopropyl alcohol before dispersing onto a holey carbon-coated $3 \mathrm{~mm}$ diameter copper electron microscope grid. We used a JEOL 4000EX transmission electron microscope which was operated 


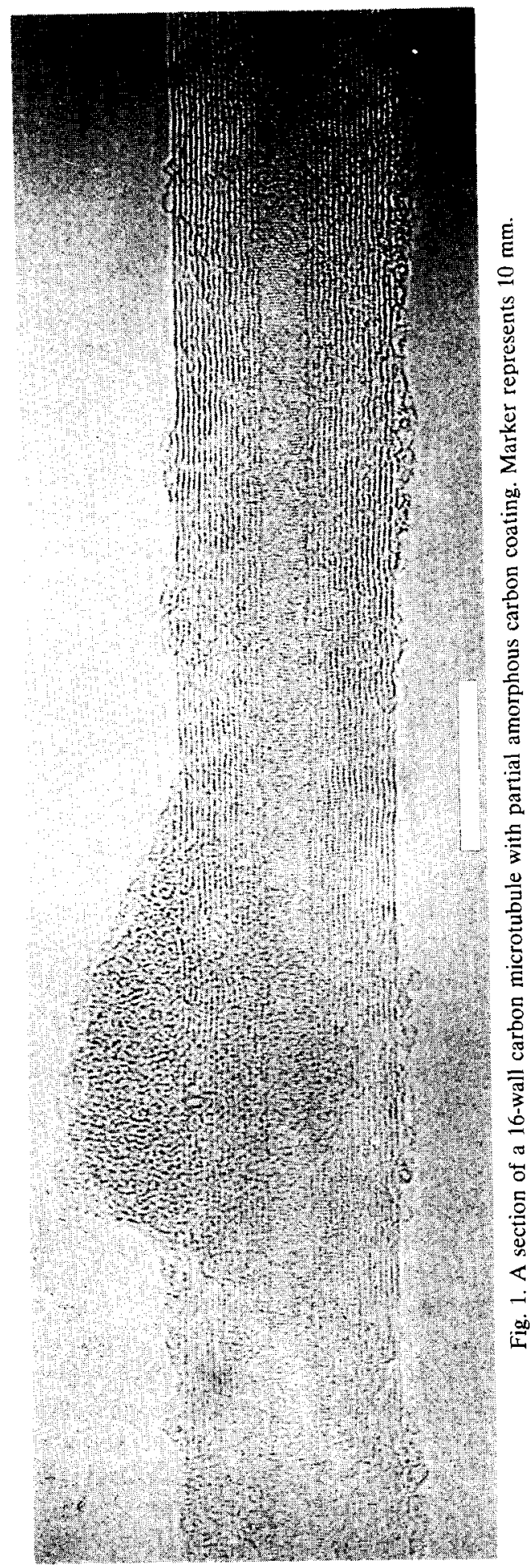


at a beam area of $7 \times 10^{-10} \mathrm{~cm}^{2}$ and a beam current of about $17 \mathrm{pA} / \mathrm{cm}^{2}$. To minimize radiation damage the micrographs presented here were obtained within 5 min of beam exposure to fresh sample areas. We saw many nested polyhedra (graphitic "onions") and microtubules interspersed with folded graphitic sheets. All of the microtubules were thick walled, containing more than a dozen layers and all fiber ends observed possessed endcaps.

A representative microtubule whose core region was resolved particularly well is displayed in Fig. 1. The fiber, composed of $16+$ concentric graphitic sheets enclosing a $3.2 \mathrm{~nm}$ core region, is virtually straight, having no visible bow over a 200 $\mathrm{nm}$ length. Amorphous carbon black is visible attached to the fiber surface. The fringes with high contrast running parallel to the fiber direction are graphitic $\{0002\}$ fiber walls viewed edge on. There are also slightly curved fringes of lower contrast which appear as closely nested parabolas. Similar parabolic contrast features were reported by Zhang et al. for carbon microtubules [5], and by Yada for chrysotile asbestos fibers [6]. Chrysotile fibers are observed in two forms, cylindrical and spiral. The spiral configuration is characterized by outer and inner walls which terminate abruptly and present distinct diagonal or radial lines across the fiber image. No spiral step planes are reported in Ref. [5] and none were seen here, suggesting that our microtubule is of concentric rather than scroll construction. This also agrees with the image simulation results (type A) of Zhang et al. [5] which support the concentric tubule interpretation.

The basal plane is best imaged in the core region where atomic alignment normal to the electron beam is nearly exact. We can clearly resolve the graphite $\{10 \overline{1} 0\}$ planes in Fig. 2a, which is an enlargement of Fig. 1. These planes consist of a bilayer of carbon atoms spaced 0.142 $\mathrm{nm}$ apart and positioned alternately above and below an average position. Due to the resolution limitations of the instrument $(0.175 \mathrm{~nm})$, we are unable to resolve the individual bilayers (sawteeth), and observe the "averaged out" positions as an hexagonal array rotated $30^{\circ}$ with respect to the actual lattice (cf. lattice schematic of graphite shown in Fig. 2b). If the $\{11 \overline{2} 0\}$ planes were prependicular to the fiber axis, the observed hexagonal array would be further rotated by $30^{\circ}$. The image contrast is consistent with an A-A stacking sequence of cylindrical layers.

The $\{10 \overline{10}\}$ planes, known to be $0.2131 \mathrm{~nm}$ apart, are used for calibration. Line density profiles of the $\{10 \overline{1} 0\}$ planes and the $(0002)$ walls were obtained from a digitized image of our micrograph. Irrespective of fiber pitch, radial profiles taken over $7.5 \mathrm{~nm}$ (300 pixel) lengths of the fiber give an average (0002) wall spacing of $0.375 \mathrm{~nm}$, accurate to $1 \%$ with a non-uniformity of $<5 \%( \pm 1$ pixel $)$. This result is insensitive to profile length until it approaches a single line profile. The $0.375 \mathrm{~nm}$ spacing is considerably larger than the $0.335 \mathrm{~nm}$ of single crystal graphite, and is greater even than the $0.344 \mathrm{~nm}$ spacing of fully turbostratic graphite [7]. Assuming the zero helicity configuration of Fig. 2b, simple geometry indicates that each successive cylindrical wall has on average 9.6 additional hexagons in its circumference. Interestingly, this is exactly one more hexagon per cylinder than the 8.6 hexagons needed for the non helical fibers of graphitic spacing found by Zhang et al. [5] and others. Half-integer hexagon counts imply that every second cylinder actually possesses a small chirality whose angle is determined from a one-hexagon row shift required for lattice continuity on closure. Thus, our inmost cylinder of diameter 3.17 $\mathrm{nm}$ is about 40.5 hexagons in circumference with an helicity of $1.2^{\circ}$, the next cylinder is non-helical with a 50.0 hexagon circumference, the 3 rd has about 59.5 hexagons with $0.8^{\circ}$ helicity, etc. (A detailed discussion of the situation can be found on p. 372 of Ref. [5].

These small chiral differences are not resolved in our micrograph. On the other hand, further inspection of Fig. 2a reveals faint superlattice hexagons overlying the core. As described by Ge and Sattler [8], these are the result of moiré interference patterns between fiber basal planes slightly misoriented with respect to one another. The faint contrast implies that only a few layers are misoriented, while the superlattice hexagon spacing indicates a misorientation of $\sim 6^{\circ}$. Since a moiré pattern can derive from the entire fiber 


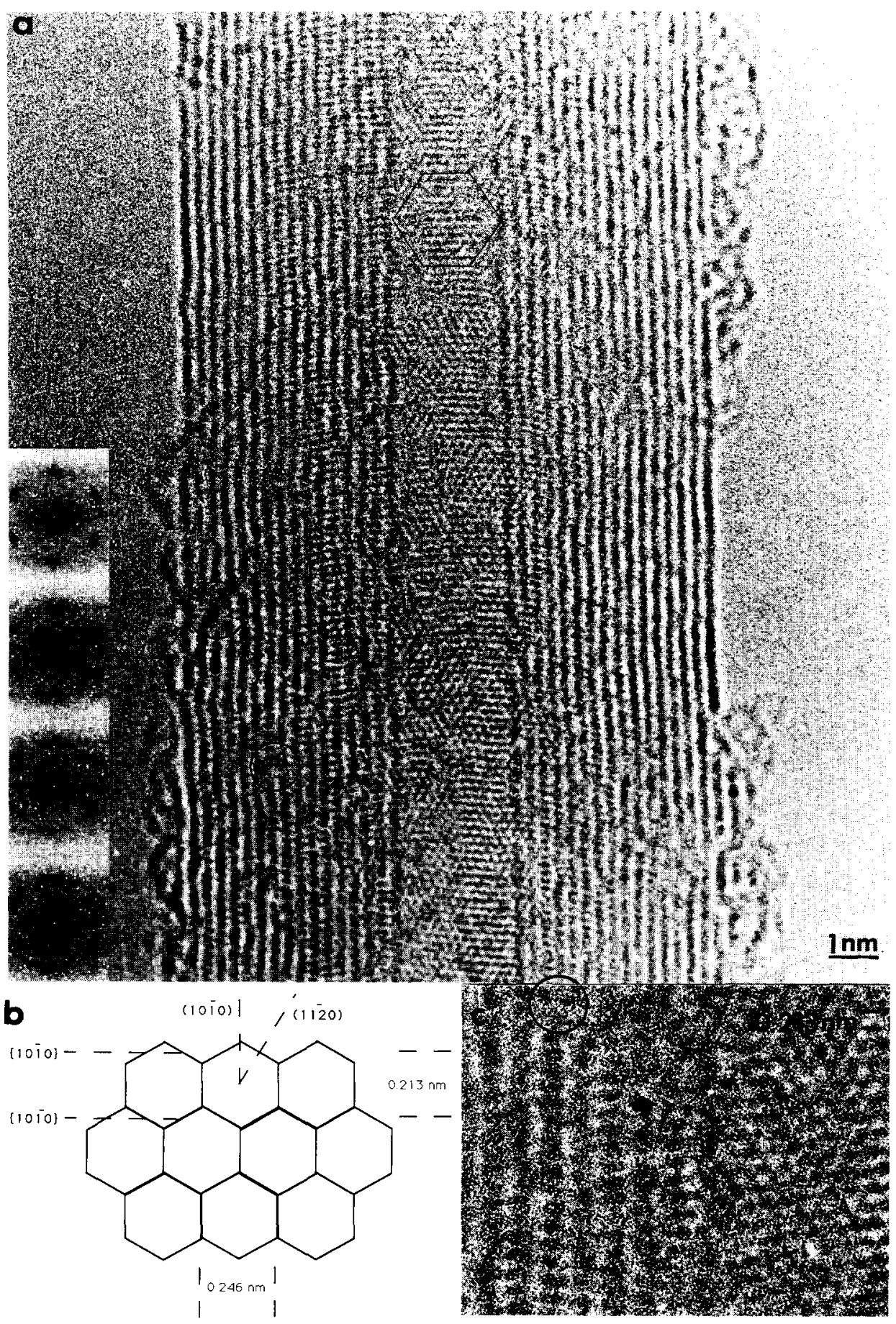

Fig. 2. (a) Enlargement of one microtubule section that displays local lattice defects in the core (open arrows) and sidewalls (circles). Hexagon drawn in the core delineates a superlattice figure (print has a distorted aspect ratio). Insets show local area FFTs generated on-axis at the respective inset positions. (b) Schematic drawing of a graphite lattice showing relevant directions and distances. The (1010) direction is along the fiber axis, in agreement with our non-helical fiber interpretation. (c) Solid arrow points to the dome shaped endcap of an embedded microtubule. Open arrows and circle indicate similiar features as in(a). 
[9], a $3^{\circ}$ top-bottom misorientation of only a wall or two could cause the effect.

The presence of basal plane atomic structure contrast throughout the core region and beyond requires nearly exact atomic alignment within the 5-10 $\mathrm{nm}$ nearest the surface [10]. However, perfect basal plane alignment is ruled out in view of the inability of curved graphitic sheets of different radii to possess a stacking sequence in the direction of curvature (although stacking in the axial direction is permissible). Tangential shear strain can be minimized and approximate radial stacking can be realized if the additional hexagons in each successive cylinder are inserted at equally spaced intervals above previously inserted hexagons to form V-shaped "interfacial dislocations" [5]. This arrangement keeps all overlaying hexagons in sequence when viewed by a parallel electron beam, thus permitting multiple $\{10 \overline{1} 0\}$ sets to be seen to off-axis positions. Density functional calculations by Charlier and Michenaud [11] predict that at room temperature, free rotation and translation of concentric graphitic cylinders will occur along their mutual $(11 \overline{2} 0)$ axis for small, $0.339 \mathrm{~nm}$ spaced double wall microtubules of limited length. The observed stabilization of actual tubule walls is likely linked to pinning by end caps, defects, and V-shaped interfacial dislocation.

Axial shear strain appears to create local helicity in the fiber. Near the center of Fig. 2a, for instance, an extra $\{10 \overline{1} 0\}$ line occurs (top open arrow). There is also an area of lattice mismatch directly outward from the line defect (large circle). The fiber readjusts from a non-helical pitch (top half of Fig. 2a) to about a $-6^{\circ}$ pitch to the left of the fiber axis, and to a $+3^{\circ}$ helicity to the right. About $4 \mathrm{~nm}$ farther down the fiber another dislocation lying on $\{10 \overline{10} 0$ (open arrow) and two on $\{0001\}$ (small circle) convert the left fiber pitch to $+3^{\circ}$ as well. Local area fast Fourier transform power spectra (FFT) inserted in Fig. 2a are taken from four different positions centered on the core at the inset positions. In all cases, we observe a six-fold array, characteristic of the basal plane, along with a row of spots orthogonal to the fiber axis arising from the tubule sidewalls. Since each FFT maximum arises from a set of atomic planes that run orthogonal to the line joining it to the origin, the particular orientation of the FFT pattern corroborates our interpretation of the atomic lattice image. Note that two sets of spots are split across the fiber axis direction in the top FFTs. The symmetric splitting and the clockwise rotation of dots in the bottom FFTs agree with our observations above.

The fiber remains $+3^{\circ}$ helical for some $7 \mathrm{~nm}$, past the bottom of Fig. 2a, where we note a peculiar defect in the enlarged image of Fig. 2c. It consist of a double walled U-shaped object (solid arrow), which we believe to be a nested tubule. Surrounding this feature are discontinuities in the sidewalls (circle) and in the core (open arrow). These defects have the important effect of locally returning the fiber back to a non-helical configuration.

The above defects are only imaged in the top portion of the fiber. We have no evidence that the helical zone imaged extends to the far wall of the fiber. The observed correspondence of dislocation features at different radii, but at the same axial position (Fig. 2a), as well as the presence of an imbedded tubule constitute strong evidence for the axial growth of our fiber. Presumably, fiber defects were formed during growth. We also observed random wall defect formation in situ under continued electron beam irradiation. After an additional half hour of irradiation at double the original beam current, loss of core and outer wall definition occurred [12].

Implications for the fiber's electrical character come from an extrapolation of tight binding calculations for small diameter microtubules by Hamada et al. [13]. Periodic boundary conditions around the fiber circumference only allow electron wave vectors on parallel lines in the graphite Brillouin zone (BZ). When one of these lines intersects the $\mathrm{K}$ point in the $\mathrm{BZ}$ (where the bonding and anti-bonding $\pi$ bonds are degenerate), the cylinder is a metal; otherwise, it is a semiconductor. The energy band gap is a function of the distance from the wave vector lines to the $\mathrm{K}$ point, and thus is dependent on cylinder diameter and pitch. Our large diameter microtubule will have very closely spaced BZ lines giving outer cylinder direct band gaps of less than a few tenths 
of an electron volt. Fiber segments of uniform $3^{\circ}$ helicity will have finite band gap shifts for the innermost cylinders. Strained fiber zones will have altered bands of unknown character.

The helical fiber regions surrounded by defects are under high stress. These regions could have been formed through abrupt changes in the growth environment. Since our rod was hand operated, the gap was frequently readjusted and occasionally the plasma spark had to be restarted. Although this occurred quickly while the rods were still orange hot, some atomic defects were evidently created. If so, then perhaps fiber packets having a prescribed sequence of axially varying band gaps could be obtained.

In summary, we analyzed the structure of a thick-walled carbon microtubule, resolving the six-fold symmetry of basal planes overlying the fiber core and locating restricted lengths of low helicity in an otherwise non-helical fiber. Sidewall spacings of $0.375 \mathrm{~nm}$ calibrated from the $\{10 \overline{1} 0\}$ core fringes indicate that 9.6 extra hexagons are incorporated in each successive cylinder. Structural transitions bordering segments or zones of local $3^{\circ}$ helicity are characterized by extra atomic planes and other defects, including nested subtubules. Low helicity zones should possess both axially and radially varying electron band gaps and their placement along the fiber might be prescribed during growth.
We thank J.H. Bretz for help with fiber production. M.B. was supported in part by a Research Opportunity Award from the Research Corporation and a Rackham Research Grant.

\section{References}

[1] S. Iijima, Nature 354 (1991) 56.

[2] S. Iijima, T. Ichihasha and Y. Ando, Nature 356 (1992) 776.

[3] T.W. Ebbeson and P.M. Ajayan, Nature 358 (1992) 220.

[4] D.S. Bethune et al. Nature 363 (1993) 605; S. Iijima and T. Ichihashi, Nature 363 (1993) 6.

[5] X.F. Zhang, X.B. Zhang, G. Van Tendeloo, S. Amelinckx, M. Op de Beeck and J. Van Landuyt, J. Crystal Growth 130 (1993) 368.

[6] K. Yada, Acta Cryst. A 27 (1971) 659.

[7] G.E. Bacon, Acta Cryst. 4 (1951) 558; partially turbostratic graphite spacings are 0.3408 and $0.3376 \mathrm{~nm}$.

[8] M. Ge and K. Sattler, Science 260 (1992) 515.

[9] P. Hirsch, A. Hawie, R.B. Nicholson, D.W. Pashley and M.J. Whelan, Electron Microscopy in Thin Crystals (Krieger, Malabar, FL, 1977) pp. 357-365.

[10] J.C.H. Spence, Experimental High Resolution Electron Microscopy, 2nd ed. (Oxford Univ. Press, Oxford, 1988) pp. 140-144.

[11] J.-C. Charlier and J.-P. Michenaud, Phys. Rev. Lett. 70 (1993) 1858.

[12] D. Ugarte, Nature 359 (1992) 707, describes major microtubule disruption in a fiber irradiation study using beam intensities much higher than ours.

[13] N. Hamada, S. Sawada and A. Oshiyama, Phys. Rev. Lett. 68 (1992) 1579. 